TRANSACTIONS OF THE

AMERICAN MATHEMATICAL SOCIETY

Volume 351, Number 6, Pages 2553-2567

S 0002-9947(99)02399-5

Article electronically published on February 9, 1999

\title{
THERMAL CAPACITY ESTIMATES ON THE ALLEN-CAHN EQUATION
}

\author{
RICHARD B. SOWERS AND JANG-MEI WU
}

\begin{abstract}
We consider the Allen-Cahn equation in a well-known scaling regime which gives motion by mean curvature. A well-known transformation of this PDE, using its standing wave, yields a PDE the solution of which is approximately the distance function to an interface moving by mean curvature. We give bounds on this last fact in terms of thermal capacity. Our techniques hinge upon the analysis of a certain semimartingale associated with a certain PDE (the PDE for the approximate distance function) and an analogue of some results by Bañuelos and Øksendal relating lifetimes of diffusions to exterior capacities.
\end{abstract}

\section{INTRODUCTION}

One way of constructing the evolution of a surface by mean curvature is through the Allen-Cahn equation

$$
\frac{\partial u^{\varepsilon}}{\partial t}=\frac{1}{2} \Delta u^{\varepsilon}+\frac{1}{\varepsilon^{2}} f\left(u^{\varepsilon}\right), \quad t>0 .
$$

Here $f: \mathbb{R} \rightarrow \mathbb{R}$ is $C^{\infty}$, odd, zero at $-1,0$, and 1 , positive on $(0,1)$, and it has a nonzero derivative at $0,-1$, and 1 . We assume that the initial data $u^{\varepsilon}(0, \cdot)$ takes on both positive and negative values.

In [ESS], Evans, Soner, and Souganidis (see also [BSS]) proved that, if we define

$$
\Gamma_{t}^{\varepsilon} \stackrel{\text { def }}{=}\left\{x \in \mathbb{R}^{d}: u^{\varepsilon}(t, x)=0\right\}
$$

and $\Gamma_{t} \stackrel{\text { def }}{=} \lim _{\varepsilon \rightarrow 0} \Gamma_{t}^{\varepsilon}$, then $\left\{\Gamma_{t}: t \geq 0\right\}$ moves by mean curvature, this being understood via the mean curvature equations (see [CGG] and [ESI]-[ESIV]). They considered initial data of the form

$$
u^{\varepsilon}(0, x)=q\left(\operatorname{dist}\left(x, \Gamma_{0}\right) / \varepsilon\right), \quad x \in \mathbb{R}^{d},
$$

where $\Gamma_{0}$ is the smooth boundary of a bounded, connected, and open subset $U$ of $\mathbb{R}^{d}$ (of course $\Gamma_{0}^{\varepsilon}=\Gamma_{0}$ ), $\operatorname{dist}\left(x, \Gamma_{0}\right)$ is the signed distance from $x$ to $\Gamma_{0}$ (being positive if $x \in U^{\circ}$ and negative if $\left.x \notin \bar{U}\right)$, and $q: \mathbb{R} \rightarrow \mathbb{R}$ is the standing wave for

Received by the editors October 20, 1997 and, in revised form, April 28, 1998.

1991 Mathematics Subject Classification. Primary 31B35, 35K57, 60J45.

Key words and phrases. Allen-Cahn equation, mean curvature, thermal capacity.

The work of R. S. was supported by NSF Grants DMS 96-26398 and DMS 96-15877 and the Research Board of the University of Illinois at Urbana-Champaign.

The work of J.-M. W. was supported by NSF Grant DMS 97-05227. 
(1); i.e., the unique odd solution of

$$
\frac{1}{2} \ddot{q}+f(q)=0, \quad x \in \mathbb{R},
$$

such that $q(0)=0$ and $\lim _{x \rightarrow \pm \infty} q(x)= \pm 1$.

The proofs in [BSS] and [ESS] relied upon an analysis of the quantity

$$
w^{\varepsilon}(t, x) \stackrel{\text { def }}{=} \varepsilon q^{-1}\left(u^{\varepsilon}(t, x)\right), \quad t \geq 0, x \in \mathbb{R}^{d} .
$$

It is easy to see that $w^{\varepsilon}$ satisfies the PDE

$$
\frac{\partial w^{\varepsilon}}{\partial t}=\frac{1}{2} \Delta w^{\varepsilon}+\frac{1}{\varepsilon} F\left(w^{\varepsilon} / \varepsilon\right)\left(\left\|\nabla w^{\varepsilon}\right\|^{2}-1\right)
$$

where

$$
F(z) \stackrel{\text { def }}{=} \frac{f(q(z))}{\dot{q}(z)}, \quad z \in \mathbb{R} .
$$

The initial data (2) implies that $w^{\varepsilon}(0, x)=\operatorname{dist}\left(x, \Gamma_{0}\right)$ for all $x \in \mathbb{R}^{d}$. Matching like powers of $\varepsilon$ in (3), we first hope that in some sense $w^{\varepsilon}$ satisfies the eikonal equation; i.e.

$$
\left\|\nabla w^{\varepsilon}\right\| \approx 1
$$

as $\varepsilon$ tends to zero. Thus $w^{\varepsilon}(t, \cdot)$ is a function which is zero on $\Gamma_{t}^{\varepsilon}$ and has gradient with magnitude 1 . This more or less implies that

$$
w^{\varepsilon}(t, x) \approx \operatorname{dist}\left(x, \Gamma_{t}^{\varepsilon}\right)
$$

If this is accurate enough, then $w^{\varepsilon}$ satisfies

$$
\begin{aligned}
\frac{\partial w^{\varepsilon}}{\partial t} & \approx \frac{1}{2} \Delta w^{\varepsilon}, \\
w^{\varepsilon}(0, x) & =\operatorname{dist}\left(x, \Gamma_{0}\right), \quad t>0,
\end{aligned}
$$

for $t \geq 0$ and $x \in \Gamma_{t}^{\varepsilon}$, and this implies that $\Gamma^{\varepsilon}$ approximately moves by mean curvature.

We here would like to study (3) probabilistically with the goal of getting some bounds on (4). In particular, we will fix positive constants $a, \delta$, and $L$ and study the structure of the sets

$$
\begin{aligned}
& A_{a, L}^{\varepsilon} \stackrel{\text { def }}{=}\left\{(t, x) \in \mathbb{R}_{+} \times \mathbb{R}^{d}:\left\|\nabla w^{\varepsilon}(t, x)\right\|^{2}>1+a \text { and }\left|w^{\varepsilon}(t, x)\right|<L\right\}, \\
& B_{a, \delta, L}^{\varepsilon} \stackrel{\text { def }}{=}\left\{(t, x) \in \mathbb{R}_{+} \times \mathbb{R}^{d}:\left\|\nabla w^{\varepsilon}(t, x)\right\|^{2}<1-a \text { and } \delta \varepsilon<\left|w^{\varepsilon}(t, x)\right|<L\right\} .
\end{aligned}
$$

These sets give some idea of the oscillations of $w^{\varepsilon}$. Although we would like to show that $A_{a, L}^{\varepsilon}$ and $B_{a, \delta, L}^{\varepsilon}$ are in some sense small, we will instead show that they have "large" complements. This will make (4) a bit more precise. Our end result is given in terms of thermal capacity (since capacity is not a Borel measure, the smallness of a set is not equivalent to the largeness of its complement). To remind ourselves what this means, define

$$
\mathfrak{g}(t, x) \stackrel{\text { def }}{=} \begin{cases}(2 \pi t)^{-d / 2} \exp \left[-\|x\|^{2} /(2 t)\right] & \text { if } t>0 \\ 0 & \text { if } t \leq 0 .\end{cases}
$$


If $K \subset \subset \mathbb{R} \times \mathbb{R}^{d}$, let $\mathcal{M}^{+}(K)$ be the collection of all nonnegative Radon measures $\mu$ on $\mathbb{R} \times \mathbb{R}^{d}$ with support in $K$; for such a $\mu$, define

$$
(\mathfrak{P} \mu)(t, x) \stackrel{\text { def }}{=} \int_{(s, y) \in \mathbb{R} \times \mathbb{R}^{d}} \mathfrak{g}(t-s, x-y) \mu(d s, d y), \quad(t, x) \in \mathbb{R} \times \mathbb{R}^{d} .
$$

Then the thermal capacity of $K$ is

$$
\operatorname{Cap}(K) \stackrel{\text { def }}{=} \sup \left\{\mu\left(\mathbb{R} \times \mathbb{R}^{d}\right): \mu \in \mathcal{M}^{+}(K) \text { and } \sup _{(t, x) \in \mathbb{R} \times \mathbb{R}^{d}}(\mathfrak{P} \mu)(t, z) \leq 1\right\} \text {. }
$$

Next, for $(t, x) \in \mathbb{R} \times \mathbb{R}^{d}$ and $\alpha>0$, define

$$
D(t, x ; \alpha) \stackrel{\text { def }}{=}\left\{(s, y) \in \mathbb{R} \times \mathbb{R}^{d}: t-\alpha \leq s \leq t,\|y-x\| \leq \sqrt{\alpha}\right\} .
$$

Then our main result is

Theorem 1. Fix positive constants $a, \delta$, and $L$. Then there are $a \beta>0$ and $a$ $\gamma>0$ such that, for all $\varepsilon>0$,

$$
\begin{array}{cl}
\frac{\operatorname{Cap}\left(D(t, x ; \beta \varepsilon) \backslash A_{a, L}^{\varepsilon}\right)}{\operatorname{Cap}(D(t, x ; \beta \varepsilon))} \geq \gamma & \text { all }(t, x) \in A_{a, L}^{\varepsilon}, \\
\frac{\operatorname{Cap}\left(D(t, x ; \beta \varepsilon) \backslash B_{a, \delta, L}^{\varepsilon}\right)}{\operatorname{Cap}(D(t, x ; \beta \varepsilon))} \geq \gamma & \text { all }(t, x) \in B_{a, \delta, L}^{\varepsilon} .
\end{array}
$$

Essentially, this means that for any $(t, x)$ in $A_{a, L}^{\varepsilon}$ (or $B_{a, \delta, L}^{\varepsilon}$ ), a "significant" portion of the thermal capacity of the small closed neighborhood $D(t, x ; \beta \varepsilon)$ of $(t, x)$ is contained in the complement of $A_{a, L}^{\varepsilon}$ (or $B_{a, \delta, L}^{\varepsilon}$ ). In fact, our arguments allow us to replace $\varepsilon$ by $\varepsilon^{2}$ if we replace $L$ by $L \varepsilon$.

Theorem 2. Fix positive constants $a, \delta$, and $L$. Then there are $a \beta>0$ and $a$ $\gamma>0$ such that, for all $\varepsilon>0$,

$$
\begin{array}{cl}
\frac{\operatorname{Cap}\left(D\left(t, x ; \beta \varepsilon^{2}\right) \backslash A_{a, L \varepsilon}^{\varepsilon}\right)}{\operatorname{Cap}\left(D\left(t, x ; \beta \varepsilon^{2}\right)\right)} \geq \gamma & \text { all }(t, x) \in A_{a, L \varepsilon}^{\varepsilon}, \\
\frac{\operatorname{Cap}\left(D\left(t, x ; \beta \varepsilon^{2}\right) \backslash B_{a, \delta, L \varepsilon}^{\varepsilon}\right)}{\operatorname{Cap}\left(D\left(t, x ; \beta \varepsilon^{2}\right)\right)} \geq \gamma & \text { all }(t, x) \in B_{a, \delta, L \varepsilon}^{\varepsilon} .
\end{array}
$$

Our basic argument is as follows. We look at the behavior of $w^{\varepsilon}$ along a (timereversed) parabolic Brownian motion; our method of study is stability theory of SDE's. We show that a parabolic Brownian motion is very likely to leave $A_{a, L}^{\varepsilon}$ and $B_{a, \delta, L}^{\varepsilon}$ quickly (by time of order $\varepsilon$ as $\varepsilon$ tends to zero). Some calculations similar to those of Bañuelos and Øksendal [BanOks] then lead to Theorems 1 and 2.

Several explanatory comments are in order. First, although the presence of $\delta$ in the definition of $B_{a, \delta, L}^{\varepsilon}$ at first looks a bit disconcerting, it is in fact natural; it excludes the identically zero solution of the PDE (3); without this, we could not hope to bound the second ratios in Theorems 1 and 2 away from zero. Second, note that our analysis does not depend on the assumption that the initial condition is of the form (2); in fact, our results will hold for any initial condition of the PDE (1). Soner ([Sonera] and [Sonerb]) has considered some other questions (via a different approach) concerning the effects of the initial condition on interface formation in Ginzburg-Landau equations. 
The connection of our work to other work is as follows. The essence of [BSS] and [ESS] is a pointwise verification of (5) and the fact that $\Gamma^{\varepsilon}$ approximately moves by mean curvature; a functional investigation of (5) is not developed. Such a functional theory is partially considered in [Illmanen], in which a Ginzburg-Landau energy measure is shown to tend to a varifold satisfying Brakke's definition of motion by mean curvature. Indeed, if one were to try to prove the results of our paper by analytical means, a reasonable place to start would be Ilmanen's paper and some of the Huisken-monotonicity arguments therein.

\section{A stochastic Differential EQUATION}

Let $\Omega \stackrel{\text { def }}{=} C\left([0, \infty) ; \mathbb{R}^{d}\right)$. Let $W$ be the coordinate process on $\Omega$, and let $\mathcal{F} \stackrel{\text { def }}{=}$ $\sigma\left\{W_{s} ; s \geq 0\right\}$. For each $x \in \mathbb{R}^{d}$, let $\mathbb{P}_{x}$ be Wiener measure on $(\Omega, \mathcal{F})$ such that $\mathbb{P}_{x}\left\{W_{0}=x\right\}=1$; let $\mathbb{E}_{x}$ be the associated expectation operator (we will later consider parabolic Brownian motion, but for the moment let's keep things as simple as possible). Fix $T>0$ and define

$$
Z_{s}^{\varepsilon} \stackrel{\text { def }}{=} w^{\varepsilon}\left(T-s, W_{s}\right), \quad 0 \leq s \leq T,
$$

for every $\varepsilon>0$. Then it is easy to see that, under $\mathbb{P}_{x}$,

$$
d Z_{s}^{\varepsilon}=d M_{s}^{\varepsilon}+\frac{1}{\varepsilon} F\left(Z_{s}^{\varepsilon} / \varepsilon\right)\left\{d\left\langle M^{\varepsilon}\right\rangle_{s}-d s\right\}, \quad 0 \leq s \leq T,
$$

where

$$
M_{s}^{\varepsilon} \stackrel{\text { def }}{=} \sum_{j=1}^{d} \int_{0}^{s} \frac{\partial w^{\varepsilon}}{\partial x_{j}}\left(T-r, W_{r}^{x}\right) d W_{r}^{x, j}, \quad 0 \leq s \leq T,
$$

and where, of course,

$$
\left\langle M^{\varepsilon}\right\rangle_{s} \stackrel{\text { def }}{=} \int_{0}^{s}\left\|\nabla w^{\varepsilon}\left(T-r, W_{r}^{x}\right)\right\|^{2} d r, \quad 0 \leq s \leq T .
$$

Thus, statements about $\left\|\nabla w^{\varepsilon}\right\|$ can be translated into statements about $\left\langle M^{\varepsilon}\right\rangle$.

Let's next take a look at the function $F$. L'Hôpital's rule implies that

$$
\lim _{z \rightarrow 0} \frac{F(z)}{z}=\dot{f}(0)>0
$$

and that

$$
\lim _{z \rightarrow \infty}(F(z))^{2}=\lim _{z \rightarrow \infty} \frac{f(q(z))^{2}}{(\dot{q}(z))^{2}}=\lim _{z \rightarrow \infty} \frac{f(q(z)) \dot{f}(q(z)) \dot{q}(z)}{\dot{q}(z) \ddot{q}(z)}=-\frac{1}{2} f^{\prime}(1),
$$

or rather, since $F$ is odd,

$$
\lim _{z \rightarrow \pm \infty} F(z)= \pm \kappa
$$

where

$$
\kappa \stackrel{\text { def }}{=} \sqrt{-\frac{1}{2} f^{\prime}(1)}
$$

We now see what has to happen. For small $\varepsilon$, (6) looks like

$$
d Z_{s}^{\varepsilon} \approx \frac{\kappa}{\varepsilon} \operatorname{sign}\left(Z_{s}^{\varepsilon}\right)\left\{d\left\langle M^{\varepsilon}\right\rangle_{s}-d s\right\}
$$

If $d\left\langle M^{\varepsilon}\right\rangle_{s} \gg d s$, then $\left|Z_{s}^{\varepsilon}\right| \rightarrow \infty$ in some sense, and if $d\left\langle M^{\varepsilon}\right\rangle_{s} \ll d s$, then $\left|Z_{s}^{\varepsilon}\right| \rightarrow 0$. If we assume the initial condition $(2)$, then $Z_{T}^{\varepsilon}=\operatorname{dist}\left(W_{T}^{x}, \Gamma_{0}\right)$, so we can't have either $\left|Z_{s}^{\varepsilon}\right| \rightarrow \infty$ or $\left|Z_{s}^{\varepsilon}\right| \rightarrow 0$. Thus, we must have that $d\left\langle M^{\varepsilon}\right\rangle_{s} \approx d s$. 


\section{AN UPPER BOUND}

We first show that the complement of $A_{a, L}^{\varepsilon}$ is "large" in a certain sense; this is roughly equivalent to showing that $\left\langle M^{\varepsilon}\right\rangle$ cannot be larger than $1+a$, which is in turn equivalent to giving an upper bound on $\left\|\nabla w^{\varepsilon}\right\|$ in terms of $1+a$. Fix $(T, x) \in A_{a, L}^{\varepsilon}$ and define the random time (i.e., measurable with respect to $(\Omega, \mathcal{F})$ )

$$
\begin{aligned}
\tau_{1}^{\varepsilon} & \stackrel{\text { def }}{=} \inf \left\{s \in(0, T]:\left(T-s, W_{s}\right) \notin A_{a, L}^{\varepsilon}\right\} \\
& =\inf \left\{s \in(0, T]:\left|Z_{s}^{\varepsilon}\right| \geq L \text { or } d\left\langle M^{\varepsilon}\right\rangle_{s} / d s \geq 1+a\right\} \quad(\inf \emptyset=T) .
\end{aligned}
$$

We will show that $\tau_{1}^{\varepsilon}$ is small and that the process $\left(T-s, W_{s}\right)$ should hit the complement of $A_{a, L}^{\varepsilon}$ very quickly. In particular, we will show that there is a $K>0$ such that

$$
\mathbb{E}_{x}\left[\tau_{1}^{\varepsilon}\right] \leq K \varepsilon
$$

where $K$ does not depend on $T, x$, or $\varepsilon$. To visually ease our arguments, we will drop the dependence on $x$ until we state the final result of this section (Proposition $3.3)$.

Our starting point is the formula

$$
\begin{array}{r}
\left|Z_{s}^{\varepsilon}\right|=\left|Z_{0}^{\varepsilon}\right|+l_{s}^{0}\left(Z^{\varepsilon}\right)+\int_{0}^{s} \operatorname{sign}\left(Z_{u}^{\varepsilon}\right) d M_{u}^{\varepsilon}+\frac{1}{\varepsilon} \int_{0}^{s} F\left(\left|Z_{u}^{\varepsilon}\right| / \varepsilon\right)\left\{d\left\langle M^{\varepsilon}\right\rangle_{u}-d u\right\}, \\
0 \leq s \leq T .
\end{array}
$$

Here $\mathfrak{l}^{0}\left(Z^{\varepsilon}\right)$ is the local time of $Z^{\varepsilon}$ at 0 .

Since $\mathfrak{l}^{0}\left(Z^{\varepsilon}\right)$ is a nondecreasing process, by definition of $\tau_{1}^{\varepsilon}$, we have that

$$
L \geq \mathbb{E}\left[\left|Z_{\tau_{1}^{\varepsilon}}^{\varepsilon}\right|\right] \geq \mathbb{E}\left[\left|Z_{0}^{\varepsilon}\right|\right]+\frac{a}{\varepsilon} \mathbb{E}\left[\int_{0}^{\tau_{1}^{\varepsilon}} F\left(\left|Z_{s}^{\varepsilon}\right| / \varepsilon\right) d s\right] \geq \frac{a}{\varepsilon} \mathbb{E}\left[\int_{0}^{\tau_{1}^{\varepsilon}} F\left(\left|Z_{s}^{\varepsilon}\right| / \varepsilon\right) d s\right] .
$$

When $Z_{s}^{\varepsilon}$ is not too close to zero, $F\left(\left|Z_{s}^{\varepsilon}\right| / \varepsilon\right) \approx \kappa$. Fix $\eta>0$ such that $F(z) \geq \kappa / 2$ whenever $z \geq \eta$. Define

$$
C_{1} \stackrel{\text { def }}{=} \sup _{0 \leq z \leq \eta} F(z)<\infty
$$

Then

$$
\int_{0}^{\tau_{1}^{\varepsilon}} F\left(\left|Z_{s}^{\varepsilon}\right| / \varepsilon\right) d s \geq \frac{\kappa}{2} \tau_{1}^{\varepsilon}-C_{1} \int_{0}^{\tau_{1}^{\varepsilon}} \chi_{\left\{\left|Z_{s}^{\varepsilon}\right| \leq \eta \varepsilon\right\}} d s
$$

and hence

$$
\mathbb{E}\left[\tau_{1}^{\varepsilon}\right] \leq \frac{\varepsilon}{a}(2 / \kappa)\left\{L+C_{1} a \varepsilon^{-1} \mathbb{E}\left[\int_{0}^{\tau_{1}^{\varepsilon}} \chi_{\left\{\left|Z_{s}^{\varepsilon}\right| \leq \eta \varepsilon\right\}} d s\right]\right\} .
$$

We now want to bound the last term from above.

Some heuristics for proceeding are in Appendix A. Define

$$
H^{\varepsilon} \stackrel{\text { def }}{=} \mathbb{E}\left[\int_{0}^{\tau_{1}^{\varepsilon}} \chi_{\left\{\left|Z_{s}^{\varepsilon}\right| \leq \eta \varepsilon\right\}} d s\right], \quad \varepsilon>0 .
$$

We now make a time change (analogous to that of (22)). Set

$$
\zeta^{\varepsilon}(s) \stackrel{\text { def }}{=} \inf \left\{r \in(0, T]:\left\langle M^{\varepsilon}\right\rangle_{r}>s\right\} \quad(\inf \emptyset=T), \quad 0 \leq s \leq\left\langle M^{\varepsilon}\right\rangle_{T} .
$$


Then there is a Brownian motion $B^{\varepsilon}$ (possibly on an augmented probability triple) such that

$$
B_{s}^{\varepsilon} \stackrel{\text { def }}{=} M_{\zeta^{\varepsilon}(s)}^{\varepsilon}, \quad 0 \leq s \leq\left\langle M^{\varepsilon}\right\rangle_{T}
$$

Now define

$$
\hat{Z}_{s}^{\varepsilon} \stackrel{\text { def }}{=} \varepsilon^{-1} Z_{\zeta^{\varepsilon}\left(s \varepsilon^{2}\right)}^{\varepsilon}, \quad 0 \leq s \leq \varepsilon^{-2}\left\langle M^{\varepsilon}\right\rangle_{T},
$$

and the Brownian motion

$$
\hat{B}_{s}^{\varepsilon} \stackrel{\text { def }}{=} \varepsilon^{-1} B_{s \varepsilon^{2}}^{\varepsilon}=\varepsilon^{-1} M_{\zeta^{\varepsilon}\left(s \varepsilon^{2}\right)}^{\varepsilon}, \quad s \geq 0 .
$$

Then

$$
\hat{Z}_{s}^{\varepsilon}=\varepsilon^{-1} Z_{0}^{\varepsilon}+\hat{B}_{s}^{\varepsilon}+\int_{0}^{s} F\left(\hat{Z}_{r}^{\varepsilon}\right)\left(\frac{\left\langle\dot{M}^{\varepsilon}\right\rangle_{\zeta^{\varepsilon}\left(r \varepsilon^{2}\right)}-1}{\left\langle\dot{M}^{\varepsilon}\right\rangle_{\zeta^{\varepsilon}\left(r \varepsilon^{2}\right)}}\right) d r, \quad 0 \leq s \leq \varepsilon^{-2}\left\langle M^{\varepsilon}\right\rangle_{T}
$$

and

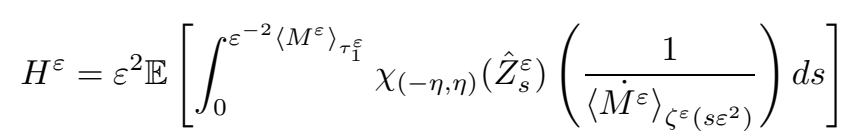

(in this last expression, we used the fact that $\zeta^{\varepsilon}$ and $\left\langle M^{\varepsilon}\right\rangle$ are inverses of each other). For $0 \leq s \leq \varepsilon^{-2}\left\langle M^{\varepsilon}\right\rangle_{T}$, we have that $\zeta^{\varepsilon}\left(s \varepsilon^{2}\right) \leq \tau_{1}^{\varepsilon}$, in which case $\left\langle\dot{M}^{\varepsilon}\right\rangle_{\zeta^{\varepsilon}\left(s \varepsilon^{2}\right)} \geq$ $1+a$. Thus, for $0 \leq s \leq \varepsilon^{-2}\left\langle M^{\varepsilon}\right\rangle_{\tau_{1}^{\varepsilon}}, \hat{Z}_{s}^{\varepsilon}=Y_{s}^{\varepsilon}$, where $Y^{\varepsilon}$ is the solution of the stochastic integral equation

$$
\begin{array}{r}
Y_{s}^{\varepsilon}=\varepsilon^{-1} Z_{0}^{\varepsilon}+\hat{B}_{s}^{\varepsilon}+\int_{0}^{s} F\left(\hat{Y}_{r}^{\varepsilon}\right)\left(1-\min \left\{\frac{1}{\left\langle\dot{M}^{\varepsilon}\right\rangle_{\zeta^{\varepsilon}\left(r \varepsilon^{2}\right)}}, \frac{1}{1+a}\right\}\right) d r \\
0 \leq s \leq \varepsilon^{-2}\left\langle M^{\varepsilon}\right\rangle_{T},
\end{array}
$$

and

$$
H^{\varepsilon} \leq \frac{\varepsilon^{2}}{1+a} \mathbb{E}\left[\int_{0}^{\infty} \chi_{(-\eta, \eta)}\left(Y_{s}^{\varepsilon}\right) d s\right]
$$

(this is the analogue of (24)).

We will now roughly follow some standard calculations involving transience (see [Stroock, Chapter 7]; these calculations will be analogous to showing transience of (23) and then showing that $G \chi_{(-\eta, \eta)}$ on the right side of $(24)$ is bounded). To simplify our notation, we will temporarily drop the superscript $\varepsilon$.

Set now $\rho_{0} \stackrel{\text { def }}{=} 0$ and

$$
\begin{aligned}
& \sigma_{n} \stackrel{\text { def }}{=} \inf \left\{t>\rho_{n-1}: Y_{s} \in[-\eta, \eta]\right\}, \\
& \rho_{n} \stackrel{\text { def }}{=} \inf \left\{t>\sigma_{n}: Y_{s} \notin[-2 \eta, 2 \eta]\right\},
\end{aligned}
$$

Then

$$
\begin{aligned}
\mathbb{E}\left[\int_{0}^{\infty} \chi_{(-\eta, \eta)}\left(Y_{s}\right) d s\right] & =\mathbb{E}\left[\sum_{j=1}^{\infty} \int_{\sigma_{n}}^{\rho_{n}} \chi_{(-\eta, \eta)}\left(Y_{s}\right) d s\right] \\
& \leq \sum_{j=1}^{\infty} \mathbb{E}\left[\mathbb{E}\left[\rho_{n}-\sigma_{n} \mid \mathcal{F}_{\sigma_{n}}\right] \chi_{\left\{\sigma_{n}<\infty\right\}}\right]
\end{aligned}
$$


where $\left\{\mathcal{F}_{t} ; t \geq 0\right\}$ is the filtration defined as

$$
\mathcal{F}_{t} \stackrel{\text { def }}{=} \mathcal{W}_{\zeta^{\varepsilon}\left(t \varepsilon^{2}\right)}, \quad t \geq 0,
$$

with $\left\{\mathcal{W}_{t} ; t \geq 0\right\}$ being the filtration generated by the original Wiener process $W$.

We now need the first of two claims, which we will prove later in Appendix B.

Lemma 3.1. For all $n \geq 1$,

$$
\mathbb{E}\left[\rho_{n}-\sigma_{n} \mid \mathcal{F}_{\sigma_{n}}\right] \leq 3 \eta^{2}
$$

on $\left\{\sigma_{n}<\infty\right\}$.

Thus,

$$
\mathbb{E}\left[\int_{0}^{\infty} \chi_{(-\eta, \eta)}\left(Y_{s}\right) d s\right] \leq 3 \eta^{2} \sum_{j=1}^{\infty} \mathbb{P}\left\{\sigma_{n}<\infty\right\}
$$

Note that, for $n \geq 1$,

$$
\begin{aligned}
\mathbb{P}\left\{\sigma_{n+1}<\infty\right\} & =\mathbb{P}\left\{\sigma_{n}<\infty, \rho_{n}<\infty, \sigma_{n+1}-\rho_{n}<\infty\right\} \\
& \leq \mathbb{E}\left[\mathbb{P}\left\{\sigma_{n+1}-\rho_{n}<\infty \mid \mathcal{F}_{\rho_{n}}\right\} \chi_{\left\{\rho_{n}<\infty\right\}} \chi_{\left\{\sigma_{n}<\infty\right\}}\right] .
\end{aligned}
$$

The second lemma we need is

Lemma 3.2. There is a constant $\alpha \in(0,1)$ (which depends upon a but not $\varepsilon$ ) such that

$$
\mathbb{P}\left\{\sigma_{n+1}-\rho_{n}<\infty \mid \mathcal{F}_{\rho_{n}}\right\} \chi_{\left\{\rho_{n}<\infty\right\}} \leq \alpha
$$

for all $n \geq 1$.

Thus, for any $n \geq 1$, we know that $\mathbb{P}\left\{\sigma_{n}<\infty\right\} \leq \alpha^{n-1}$, which immediately implies that

$$
H^{\varepsilon} \leq \varepsilon^{2} \frac{3 \eta^{2}}{(1+a)(1-\alpha)}
$$

for all $\varepsilon>0$.

Thus, we get

Proposition 3.3. There is a constant $K$ (which depends upon a and $L$ but not on $\varepsilon, T$, or $x)$ such that

$$
\mathbb{E}_{x}\left[\tau_{1}^{\varepsilon}\right] \leq \varepsilon K
$$

Proof. In fact, we have that

$$
\mathbb{E}_{x}\left[\tau_{1}^{\varepsilon}\right] \leq \varepsilon\left(\frac{2}{\kappa a}\right)\left(L+\varepsilon \frac{3 C_{1} a \eta^{2}}{(1+a)(1-\alpha)}\right) .
$$

This implies (9). 


\section{A LOWER BOUND}

We next show that the complement of $B_{a, \delta, L}^{\varepsilon}$ is "large", i.e., that

$$
\begin{aligned}
\tau_{2}^{\varepsilon} \stackrel{\text { def }}{=} \inf \left\{s \in(0, T]:\left(T-s, W_{s}\right) \notin B_{a, \delta, L}^{\varepsilon}\right\} & \\
& =\inf \left\{s \in(0, T]:\left|Z_{s}^{\varepsilon}\right| \notin(\delta \varepsilon, L) \text { or } d\left\langle M^{\varepsilon}\right\rangle_{s} / d s \leq 1-a\right\} \quad(\inf \emptyset=T)
\end{aligned}
$$

is small (which in turn gives a lower bound on $\left\|\nabla w^{\varepsilon}\right\|$ in terms of $1-a$ ). We will show that there is a $K>0$ which does not depend on $T, x$, or $\varepsilon$ such that

$$
\mathbb{E}_{x}\left[\tau_{2}^{\varepsilon}\right] \leq K \varepsilon
$$

Define

$$
C_{2} \stackrel{\text { def }}{=} \inf _{|z| \geq \delta}|F(z)|>0
$$

(here is where we need that $\delta>0$; if we would set $\delta$ to zero, then $C_{2}$ would also be zero). Since we are assuming that $(T, x) \in B_{a, \delta, L}^{\varepsilon}$, either $Z_{0}^{\varepsilon} \geq \delta$ or $Z_{0}^{\varepsilon} \leq-\delta$. Assume the former; the calculations for the latter are essentially the same. Now we have that

$$
\delta \varepsilon \leq \mathbb{E}_{x}\left[Z_{\tau_{2}^{\varepsilon}}^{\varepsilon}\right] \leq \mathbb{E}_{x}\left[Z_{0}^{\varepsilon}\right]-\frac{a}{\varepsilon} \mathbb{E}_{x}\left[\int_{0}^{\tau_{2}^{\varepsilon}} F\left(Z_{s}^{\varepsilon} / \varepsilon\right) d s\right] \leq L-\frac{a}{\varepsilon} C_{2} \mathbb{E}_{x}\left[\tau_{2}^{\varepsilon}\right] .
$$

Thus

$$
\mathbb{E}_{x}\left[\tau_{2}^{\varepsilon}\right] \leq \varepsilon \frac{L-\delta \varepsilon}{a C_{2}} .
$$

Proposition 4.1. There is a constant $K$ (which depends upon a but not on $\varepsilon, T$, or $x)$ such that

$$
\mathbb{E}_{x}\left[\tau_{2}^{\varepsilon}\right] \leq \varepsilon K
$$

\section{Some CAPACity ARguments}

The content of Propositions 3.3 and 4.1 is that $s \mapsto\left(T-s, W_{s}\right)$ leaves $A_{a, L}^{\varepsilon}$ and $B_{a, \delta, L}^{\varepsilon}$ quickly. We now convert this into Theorems 1 and 2 .

First, we need to put our problem into the framework of Markov processes; of course $s \mapsto\left(T-s, W_{s}\right)$ is a Markov process. Define

$$
\begin{aligned}
\left(\mathbf{P}_{s} f\right)((T, x)) & \stackrel{\text { def }}{=} \int_{z \in \mathbb{R}^{d}} f((T-s, z)) \mathfrak{g}(s, x-z) d z, \\
& s \geq 0, f \in C^{\infty}\left(\mathbb{R} \times \mathbb{R}^{d}\right),(T, x) \in \mathbb{R} \times \mathbb{R}^{d} .
\end{aligned}
$$

Then $\left\{\mathbf{P}_{s}, s \geq 0\right\}$ is a semigroup on $C^{\infty}\left(\mathbb{R} \times \mathbb{R}^{d}\right)$. Henceforth, we will start to use tildes to emphasize that we are working with parabolic Wiener measure rather than ordinary Wiener measure. Define now $\tilde{\Omega} \stackrel{\text { def }}{=} C\left(\mathbb{R}_{+} ; \mathbb{R} \times \mathbb{R}^{d}\right)$. Let $\tilde{X}$ be the coordinate process on $\tilde{\Omega}$, and let $\tilde{\mathcal{F}} \stackrel{\text { def }}{=} \sigma\left\{\tilde{X}_{s} ; s \geq 0\right\}$. For each $(T, x) \in \mathbb{R} \times \mathbb{R}^{d}$, let $\tilde{\mathbb{P}}_{T, x}$ be the unique measure on $(\tilde{\Omega}, \tilde{\mathcal{F}})$ under which $\tilde{X}$ is a Markov process with transition semigroup $\left\{\mathbf{P}_{s}: s \geq 0\right\}$ and such that $\tilde{\mathbb{P}}_{T, x}\left\{\tilde{X}_{0}=(T, x)\right\}=1$; let $\tilde{\mathbb{E}}_{T, x}$ 
be the associated expectation operator. Note that the $\mathbb{P}_{T, x}-$ law of $\tilde{X}$ is the same as the $\mathbb{P}_{x}$-law of the process $s \mapsto\left(T-s, W_{s}\right)$. Define

$$
\begin{gathered}
\tilde{\tau}_{1, a, L}^{\varepsilon} \stackrel{\text { def }}{=} \inf \left\{s \geq 0: \tilde{X}_{s} \notin A_{a, L}^{\varepsilon}\right\}, \\
\tilde{\tau}_{2, a, L, \delta}^{\varepsilon} \stackrel{\text { def }}{=} \inf \left\{s \geq 0: \tilde{X}_{s} \notin B_{a, \delta, L}^{\varepsilon}\right\} ;
\end{gathered}
$$

then Propositions 3.3 and 4.1 say that, for some $K>0$,

$$
\begin{gathered}
\sup _{(T, x) \in A_{a, L}^{\varepsilon}} \tilde{\mathbb{E}}_{T, x}\left[\tilde{\tau}_{1, a, L}^{\varepsilon}\right] \leq K \varepsilon, \\
\sup _{(T, x) \in B_{a, \delta, L}^{\varepsilon}} \tilde{\mathbb{E}}_{T, x}\left[\tilde{\tau}_{2, a, \delta, L}^{\varepsilon}\right] \leq K \varepsilon .
\end{gathered}
$$

In a moment, we will prove a result similar to one of Bañuelos and Øksendal [BanOks] (related results appear in [HayPomm], [Mueller], and [Stegenga]) which related the lifetime of a Brownian motion in a domain to the exterior capacities of the domain. First, however, let's recall some aspects of thermal capacity (see [Chung, Ch. 5], [Hunt], [Stroock, Ch. VIII], and [Watson]). Recall the notation of $\S 1$.

Fix a compact subset $K$ of $\mathbb{R} \times \mathbb{R}^{d}$. Define

$$
\begin{aligned}
p^{K}((T, x)) & \stackrel{\text { def }}{=} \tilde{\mathbb{P}}_{T, x}\left\{\tilde{X}_{s} \in K \text { for some } s>0\right\} \\
& =\mathbb{P}_{x}\left\{\left(T-s, W_{s}\right) \in K \text { for some } s>0\right\} .
\end{aligned}
$$

Then $p^{K}=\mathfrak{P} \mu^{K}$ for some $\mu \in \mathcal{M}^{+}(K)$, and $\operatorname{Cap}(K)=\mu^{K}(K)$. Let's now introduce some parabolic scaling. For $\alpha>0$, define $\mathbf{S}^{\alpha}: \mathbb{R} \times \mathbb{R}^{d} \rightarrow \mathbb{R} \times \mathbb{R}^{d}$ as

$$
\mathbf{S}^{\alpha}((t, x)) \stackrel{\text { def }}{=}(t \alpha, x \sqrt{\alpha}), \quad(t, x) \in \mathbb{R} \times \mathbb{R}^{d} .
$$

Noting that $\mathfrak{g} \circ \mathbf{S}^{\alpha}=\alpha^{-d / 2} \mathfrak{g}$, we have that

$$
\operatorname{Cap}\left(\mathbf{S}^{\alpha}(K)\right)=\alpha^{d / 2} \operatorname{Cap}(K)
$$

for any compact subset $K$ of $\mathbb{R} \times \mathbb{R}^{d}$ and any $\alpha>0$.

Next, we start to prove the analogue of the Bañuelos- Øksendal result. For any open subset $G$ of $\mathbb{R} \times \mathbb{R}^{d}$, define an $(\tilde{\Omega}, \tilde{\mathcal{F}})$-random time

$$
\tilde{\sigma}_{G} \stackrel{\text { def }}{=} \inf \left\{s>0: \tilde{X}_{s} \notin G\right\} .
$$

The general problem is now as follows: we have a set $G$ (i.e., either $A_{a, L}^{\varepsilon}$ or $B_{a, \delta, L}^{\varepsilon}$ ) such that

$$
a \stackrel{\text { def }}{=} \sup _{(T, x) \in G} \tilde{\mathbb{E}}_{T, x}\left[\tilde{\sigma}_{G}\right]<\infty
$$

and we want to get lower bounds (in terms of $a$ and the dimension $d$ ) on exterior thermal capacities of $G$.

First, let's assume that $(0,0) \in G$ and consider some "boxes" in $\mathbb{R} \times \mathbb{R}^{d}$. For $l>0$, define the sets

$$
\begin{gathered}
D(l) \stackrel{\text { def }}{=}\left\{(s, y) \in \mathbb{R} \times \mathbb{R}^{d}:-l \leq s \leq 0,\|y\| \leq \sqrt{l}\right\}, \\
D^{b o t}(l) \stackrel{\text { def }}{=} D(l) \cap\left(\left\{-l^{2}\right\} \times \mathbb{R}^{d}\right) .
\end{gathered}
$$

Our first result concerns the way in which $\tilde{X}$ exits a fixed $D(l)$ under $\mathbb{P}_{0,0}$ : 
Lemma 5.1. There is a $\lambda>0$ such that

$$
\tilde{\mathbb{P}}_{0,0}\left\{\tilde{X}_{\tilde{\sigma}_{D(l)}} \in D^{b o t}(l)\right\} \geq \lambda
$$

for all $l>0$.

Proof. In fact, the left-hand side of (14) is independent of $l$ due to parabolic scaling, so the inequality is actually an equality.

Next let's consider the time at which $\tilde{X}$ exits a fixed $D(l)$ under $\tilde{\mathbb{P}}_{t, x}$ for a collection of $(t, x)$ 's. For $l>0$, define

$$
B(l) \stackrel{\text { def }}{=}\{(t, x) \in D(l):-l / 2 \leq t \leq 0,\|x\| \leq \sqrt{l}-\sqrt{l+t}\} .
$$

The set $B(l)$ consists of exactly those points $(t, x)$ in $D(l) \cap\left([-l / 2,0] \times \mathbb{R}^{d}\right)$ such that $(t, x)+D(l+t) \subset D(l)$. Then we have

Lemma 5.2. For every $l>0$ and $(t, x) \in B(l)$,

$$
\tilde{\mathbb{E}}_{t, x}\left[\tilde{\sigma}_{D(l)}\right] \geq l \lambda / 2 .
$$

Proof. First, note that, for any $l>0$,

$$
\tilde{\mathbb{E}}_{0,0}\left[\tilde{\sigma}_{D(l)}\right] \geq \tilde{\mathbb{E}}_{0,0}\left[\tilde{\sigma}_{D(l)} \chi_{\left\{\tilde{X}_{\tilde{\sigma}_{D(l)}} \in D^{\text {bot }}(l)\right\}}\right] \geq l \lambda
$$

(if $\tilde{X}_{\tilde{\sigma}_{D(l)}} \in D^{b o t}(l)$, then $\tilde{\sigma}_{D(l)}=l$ ). Using the comment following (15), we thus have that

$$
\begin{aligned}
\tilde{\mathbb{E}}_{t, x}\left[\tilde{\sigma}_{D(l)}\right] & =\int_{0}^{\infty} \tilde{\mathbb{P}}_{t, x}\left\{\tilde{X}_{r} \in D(l) \text { for all } 0<r<s\right\} d s \\
& \geq \int_{0}^{\infty} \tilde{\mathbb{P}}_{t, x}\left\{\tilde{X}_{r} \in(t, x)+D(l+t) \text { for all } 0<r<s\right\} d s \\
& \geq \int_{0}^{\infty} \tilde{\mathbb{P}}_{0,0}\left\{\tilde{X}_{r} \in D(l+t) \text { for all } 0<r<s\right\} d s \\
& =\tilde{\mathbb{E}}_{0,0}\left[\tilde{\sigma}_{D(l+t)}\right] \geq(l+t) \lambda \geq l \lambda / 2 .
\end{aligned}
$$

This is what we want.

Next, let's get back to $G$ satisfying (13); note that we still are assuming that $(0,0) \in G$. Set

$$
\begin{gathered}
l \stackrel{\text { def }}{=} 4 a / \lambda, \\
\rho \stackrel{\text { def }}{=} \inf \left\{s>0: \tilde{X}_{s} \in D(l) \backslash G\right\} .
\end{gathered}
$$

Then we have

Proposition 5.3. For all $(t, x) \in B(l)$, we have

$$
\tilde{\mathbb{P}}_{t, x}\{\rho<l\} \geq \lambda / 4 \text {. }
$$

Proof. For convenience, define the $(\tilde{\Omega}, \tilde{\mathcal{F}})$-random time

$$
\eta \stackrel{\text { def }}{=} \inf \left\{s>0: \tilde{X}_{s} \notin D(l) \cap G\right\}
$$

and define the shift maps $\left\{\theta_{s}: s \geq 0\right\}$ from $\tilde{\Omega}$ to itself by

$$
\theta_{s}\left(\tilde{X}_{t}\right) \stackrel{\text { def }}{=} \tilde{X}_{t+s}, \quad t, s \geq 0 .
$$


Then

$$
\tilde{\sigma}_{D(l)}=\eta+\tilde{\sigma}_{D(l)} \circ \theta_{\eta}
$$

(separately consider the two cases where $\tilde{X}_{\eta} \in \partial D(l)$ and $\tilde{X}_{\eta} \notin \partial D(l)$ ). By our choice of $l$ and Lemma 5.2, we know that $\tilde{\mathbb{E}}_{t, x}\left[\tilde{\sigma}_{D(l)}\right] \geq 2 a$. Also, since $D(l) \cap G \subset G$, we know that $\eta \leq \tilde{\sigma}_{G}$. Third, $\tilde{\sigma}_{D(l)} \circ \theta_{\eta} \leq l$. Finally, if $\tilde{\sigma}_{D(l)} \circ \theta_{\eta}>0$, then $\tilde{X}_{\eta} \notin \partial D(l)$, in which case $\eta<l$ and $\tilde{X}_{\eta} \in D(l) \backslash G$, implying that $\rho<l$. Combining all of this, we get that

$$
2 a \leq \tilde{\mathbb{E}}_{t, x}\left[\tilde{\sigma}_{G}\right]+l \tilde{\mathbb{P}}_{t, x}\{\rho<l\} .
$$

It is now an easy step to (17).

Note that if $\rho<l$, then of course $\rho<\infty$, so $\tilde{X}$ touches $D(l) \backslash G$ in some finite time.

We now can remove the restriction that $(0,0) \in G$.

Corollary 5.4. Assume that (13) holds and define $l$ as in (16). Fix $(t, x) \in G$. Then for every $(s, y) \in B(l)+(t, x)$,

$$
\tilde{\mathbb{P}}_{s, y}\left\{\tilde{X}_{r} \in(D(l)+(t, x)) \backslash \text { G for some } r>0\right\} \geq \lambda / 4 \text {. }
$$

Proof. Translate the statement of Proposition 5.3 by $(t, x)$.

Under the same assumptions, we consequently have that

$$
\lambda / 4 \leq\left(\mathfrak{P} \mu_{(D(l)+(t, x)) \backslash G}\right)(s, y),
$$

where $\mu_{(D(l)+(t, x)) \backslash G}$ is the thermal capacitory measure of $(D(l)+(t, x)) \backslash G$. The final step is to integrate (18) in $s$ and $y$.

Proposition 5.5. Assume that (13) holds. Fix $(t, x) \in G$. Then

$$
\operatorname{Cap}((D(4 a / \lambda)+(t, x)) \backslash G) \geq\left(2^{d-1} / \lambda^{d / 2-1}\right) \mathfrak{L}^{d+1}(B(1)) a^{d / 2},
$$

where $\mathfrak{L}^{d+1}$ is $d+1$-dimensional Lebesgue measure on $\left(\mathbb{R} \times \mathbb{R}^{d}, \mathcal{B}\left(\mathbb{R} \times \mathbb{R}^{d}\right)\right)$.

Proof. Let $l$ be given by (16). Then from (18),

$$
\begin{aligned}
(\lambda / 4) & \mathfrak{L}^{d+1}(B(l)+(t, x)) \\
\leq & \int_{(s, y) \in B(l)+(t, x)} \int_{(r, w) \in \mathbb{R} \times \mathbb{R}^{d}} \mathfrak{g}(s-r, y-w) \mu_{(D(l)+(t, x)) \backslash G}(d r d w) d s d y .
\end{aligned}
$$

Note that

$$
\mathfrak{L}^{d+1}(B(l)+(t, x))=\mathfrak{L}^{d+1}(B(l))=l^{d / 2+1} \mathfrak{L}^{d+1}(B(1)) .
$$

We then use Tonelli's theorem on the right-hand side of (20) and calculate that, for any $(r, w) \in \mathbb{R} \times \mathbb{R}^{d}$,

$$
\begin{aligned}
\int_{(s, y) \in B(l)+(t, x)} \mathfrak{g}(s-r, y-w) & d s d y \\
\leq & \int_{t-l / 2<s<t}\left(\int_{y \in \mathbb{R}^{d}} \mathfrak{g}(s-r, y-w) d y\right) d s=l / 2 .
\end{aligned}
$$

Simplify this and use the definition of $l$ as in (16) to get (19). 
Corollary 5.6. There are constants $\beta>0$ and $\gamma>0$ (depending only on the dimension d) such that if (13) holds, then

$$
\frac{\operatorname{Cap}((D(\beta a)+(t, x)) \backslash G)}{\operatorname{Cap}(D(\beta a)+(t, x))} \geq \gamma .
$$

Proof. We set $\beta=4 / \lambda$ and note that, by (12),

$$
\operatorname{Cap}(D(\beta a)+(t, x))=\operatorname{Cap}(D(\beta a))=\operatorname{Cap}\left(\mathbf{S}^{a}(D(\beta))\right)=a^{d / 2} \operatorname{Cap}(D(\beta)) .
$$

This is what we want.

Thus, we finally have

Proof of Theorems 1 and 2. Almost immediate from the preceding. For Theorem 1, we use Propositions 3.3 and 4.1. For Theorem 2, we use (10) and (11).

\section{Appendix A}

Here we provide some heuristics on how to bound the expression on the right of (7). Consider the SDE

$$
\begin{aligned}
d \xi_{s}^{\beta, \varepsilon} & =\beta d W_{s}+\frac{\beta^{2}}{\varepsilon} \arctan \left(\xi_{s}^{\beta, \varepsilon} / \varepsilon\right) d s, \\
\xi_{0}^{\beta, \varepsilon} & =y
\end{aligned}
$$

and the quantity

$$
h^{\beta, \varepsilon} \stackrel{\text { def }}{=} \mathbb{E}\left[\int_{0}^{t} \chi_{(-\eta, \eta)}\left(\xi_{s}^{\beta, \varepsilon} / \varepsilon\right) d s\right] .
$$

We want to understand what happens to this quantity as $\varepsilon$ tends to zero and as $\beta$ ranges over $\left[\beta_{c}, \infty\right.$ ), where $\beta_{c}>1$ is some prespecified number (which should be compared to $\sqrt{a}$ and $\sqrt{1+a}$ ).

First, we can get rid of many of the $\beta$ 's and $\varepsilon$ 's in the dynamics of (21) by defining

$$
\hat{\xi}_{s}^{\beta, \varepsilon} \stackrel{\text { def }}{=} \varepsilon^{-1} \xi_{s \varepsilon^{2} / \beta^{2}}^{\beta, \varepsilon}, \quad s \geq 0 .
$$

Then

$$
\begin{aligned}
d \hat{\xi}_{s}^{\beta, \varepsilon} & =d W_{s}+\arctan \left(\hat{\xi}_{s}^{\beta, \varepsilon}\right) d s, \\
\hat{\xi}_{0}^{\beta, \varepsilon} & =\varepsilon^{-1} y
\end{aligned}
$$

and

$$
h^{\beta, \varepsilon}=\frac{\varepsilon^{2}}{\beta^{2}} \mathbb{E}\left[\int_{0}^{t(\beta / \varepsilon)^{2}} \chi_{(-\eta, \eta)}\left(\hat{\xi}_{s}^{\beta, \varepsilon}\right) d s\right] .
$$

One can now check that, for each $\beta \geq \beta_{c}, \hat{\xi}^{\beta, \varepsilon}$ is transient (use [KS, Proposition 5.22] or [RY, Theorem V.1.6]). Thus, we should not lose too much with the bound

$$
h^{\beta, \varepsilon} \leq \frac{\varepsilon^{2}}{\beta^{2}} \mathbb{E}\left[\int_{0}^{\infty} \chi_{(-\eta, \eta)}\left(\hat{\xi}_{s}^{\beta, \varepsilon}\right) d s\right]=\frac{\varepsilon}{\beta^{2}}\left(G \chi_{(-\eta, \eta)}\right)(y / \varepsilon),
$$

where $G$ is the Green's operator defined by the $\operatorname{SDE}(23)$. It is reasonable that $\sup _{z \in \mathbb{R}}\left(G \chi_{(-\eta, \eta)}\right)(z)<\infty$, in which case

$$
\sup _{\beta \geq \beta_{c}} \varlimsup_{\varepsilon \rightarrow 0} \varepsilon^{-1} h^{\beta, \varepsilon}=0 .
$$




\section{Appendix B}

Here we prove Lemmas 3.1 and 3.2. For convenience, let's define the mapping $b^{a}:(0, \infty) \rightarrow(0, \infty)$ as

$$
b^{a}(x) \stackrel{\text { def }}{=} 1-\min \left\{\frac{1}{x}, \frac{1}{1+a}\right\}, \quad x>0,
$$

for $a>0$.

Proof of Lemma 3.1. By Ito's formula applied to (8),

$$
\begin{aligned}
Y_{t \wedge \rho_{n}}^{2} & -Y_{t \wedge \sigma_{n}}^{2}=2 \int_{t \wedge \sigma_{n}}^{t \wedge \rho_{n}} Y_{s} d \hat{B}_{s}^{\varepsilon} \\
& +\int_{t \wedge \sigma_{n}}^{t \wedge \rho_{n}}\left\{2 Y_{s} F\left(Y_{s}\right) b^{a}\left(\left\langle\dot{M}^{\varepsilon}\right\rangle_{\zeta^{\varepsilon}\left(s \varepsilon^{2}\right)}\right)+1\right\} d s, \quad t \geq 0 .
\end{aligned}
$$

Note that $y F(y) \geq 0$ for all $y \in \mathbb{R}$, and

$$
Y_{t \wedge \rho_{n}}^{2}-Y_{t \wedge \sigma_{n}}^{2} \in\left(0,3 \eta^{2}\right)
$$

for all $t \geq 0$. Thus from (25),

$$
\mathbb{E}\left[\rho_{n} \wedge t-\sigma_{n} \wedge t \mid \mathcal{F}_{\sigma_{n}}\right] \leq 3 \eta^{2}
$$

for all $n$. Since $\rho_{n} \wedge t-\sigma_{n} \wedge t$ monotonically increases to $\rho_{n}-\sigma_{n}$ as $t$ tends to infinity, we get the result.

Proof of Lemma 3.2. First of all, note that for $n \geq 1$, if $\rho_{n}<\infty$, then $Y_{\rho_{n}} \in$ $\{-2 \eta, 2 \eta\}$. We next want to compare $Y$ to a diffusion with a drift which does not depend on $\left\langle M^{\varepsilon}\right\rangle$. We shall then analyze these diffusions via Feller's study of one-dimensional diffusions. Choose a real number $c$ such that

$$
\frac{1}{1+a} \kappa<c<\kappa
$$

and define

$$
F_{ \pm}(y) \stackrel{\text { def }}{=} F(y) \pm c, \quad y \in \mathbb{R}
$$

Then

$$
\begin{gathered}
F_{-}(y) \leq F(y) b^{a}(x) \leq F_{+}(y), \quad y \in \mathbb{R}, x>0, \\
\lim _{y \rightarrow \infty} F_{-}(y)=\kappa-c>0, \quad \text { and } \lim _{y \rightarrow-\infty} F_{+}(y)=-\kappa+c<0 .
\end{gathered}
$$

Fix now $n \geq 1$ and define $Y^{ \pm}$as the solution of the stochastic integral equation

$$
Y_{t}^{ \pm}=\mp 2 \eta+\int_{\rho_{n}}^{t} F_{ \pm}\left(Y_{s}^{ \pm}\right) d s+\left(\hat{B}_{t}^{\varepsilon}-\hat{B}_{\rho_{n}}^{\varepsilon}\right), \quad t \geq \rho_{n} .
$$

Then (by [RY, Theorem IX.3.8]), noting that $F_{ \pm}$are Lipschitz functions,

$$
\mathbb{P}\left\{Y_{t}^{+} \geq Y_{t} \text { for all } t \geq \rho_{n} \mid \mathcal{F}_{\rho_{n}}\right\}=1
$$

on the set $\left\{\rho_{n}<\infty, Y_{\rho_{n}}=-2 \eta\right\}$ and

$$
\mathbb{P}\left\{Y_{t}^{-} \leq Y_{t} \text { for all } t \geq \rho_{n} \mid \mathcal{F}_{\rho_{n}}\right\}=1
$$

on the set $\left\{\rho_{n}<\infty, Y_{\rho_{n}}=2 \eta\right\}$. Note that if $\rho_{n}<\infty$ and $Y_{\rho_{n}}=-2 \eta$, then $\sigma_{n+1}-$ $\rho_{n}<\infty$ implies that $\sup _{t \geq \rho_{n}} Y_{t} \geq-\eta$, which in turn implies that $\sup _{t \geq \rho_{n}} Y_{t}^{+} \geq-\eta$. 


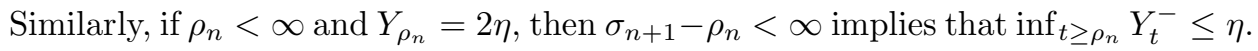
Collecting things together for a moment, we have that

$$
\begin{aligned}
\mathbb{P}\left\{\sigma_{n+1}-\rho_{n}<\infty \mid \mathcal{F}_{\rho_{n}}\right\} \chi_{\left\{\rho_{n}<\infty\right\}} \leq \mathbb{P} & \left\{\sup _{t \geq \rho_{n}} Y_{t}^{+} \geq-\eta \mid \mathcal{F}_{\rho_{n}}\right\} \chi_{\left\{\rho_{n}<\infty, Y_{\rho_{n}}=-2 \eta\right\}} \\
& +\mathbb{P}\left\{\inf _{t \geq \rho_{n}} Y_{t}^{-} \leq \eta \mid \mathcal{F}_{\rho_{n}}\right\} \chi_{\left\{\rho_{n}<\infty, Y_{\rho_{n}}=2 \eta\right\}} .
\end{aligned}
$$

We now use Feller's theory (see [KS, Section 5.5B]). Define the scale functions

$$
p^{ \pm}(x)=\int_{0}^{x} \exp \left[-2 \int_{0}^{z} F_{ \pm}(u) d u\right] d z, \quad x \in \mathbb{R},
$$

and observe that

$$
p^{+}(-\infty) \stackrel{\text { def }}{=} \lim _{x \rightarrow-\infty} p^{+}(x)>-\infty \quad \text { and } \quad p^{-}(\infty) \stackrel{\text { def }}{=} \lim _{x \rightarrow \infty} p^{-}(x)<\infty .
$$

Thus (see [KS, eq. (5.61) of Ch. 5])

$$
\mathbb{P}\left\{\sup _{t \geq \rho_{n}} Y_{t}^{+} \geq-\eta \mid \mathcal{F}_{\rho_{n}}\right\}=\frac{p^{+}(-2 \eta)-p^{+}(-\infty)}{p^{+}(-\eta)-p^{+}(-\infty)} \stackrel{\text { def }}{=} \alpha^{+}<1
$$

on the set $\left\{\rho_{n}<\infty, Y_{\rho_{n}}=-2 \eta\right\}$, and

$$
\mathbb{P}\left\{\inf _{t \geq \rho_{n}} Y_{t}^{-} \leq \eta \mid \mathcal{F}_{\rho_{n}}\right\}=\frac{p^{-}(\infty)-p^{-}(2 \eta)}{p^{-}(\infty)-p^{-}(\eta)} \stackrel{\text { def }}{=} \alpha^{-}<1
$$

on the set $\left\{\rho_{n}<\infty, Y_{\rho_{n}}=2 \eta\right\}$. Hence (for any $n \geq 1$ ),

$$
\mathbb{P}\left\{\sigma_{n+1}-\rho_{n}<\infty \mid \mathcal{F}_{\rho_{n}}\right\} \chi_{\left\{\rho_{n}<\infty\right\}} \leq \alpha \stackrel{\text { def }}{=} \max \left\{\alpha^{+}, \alpha^{-}\right\}<1 .
$$

This completes the proof.

\section{REFERENCES}

[BanOks] R. Bañuelos and B. Øksendal, Exit times for elliptic diffusions and BMO, Proceedings of the Edinburgh Mathematical Society 30 (1987), 273-287. MR 88m:58199

[BSS] G. Barles, H.M. Soner, and P.E. Souganidis, Front propagation and phase field theory, SIAM J. Control and Optimization 31 (1993), 439-469. MR 94c:35005

[CGG] Y.-G. Chen, Y. Giga, and S. Goto, Uniqueness and existence of viscosity solutions of generalized mean curvature flow equations, Proc. Japan Acad. Ser. A Math. Sci 65 (1989), 207-210. MR 91b:35049

[Chung] K. L. Chung, Lectures from Markov Processes to Brownian Motion, Springer-Verlag, New York, 1982. MR 84c:60091

[ESI] L. C. Evans and J. Spruck, Motion of level sets by mean curvature I, J. Differential Geometry 33 (1991), 635-681. MR 92h:35097

[ESII] L. C. Evans and J. Spruck, Motion of level sets by mean curvature II, Trans. Amer. Math Soc. 330 (1992), 321-332. MR 92f:58050

[ESIII] L. C. Evans and J. Spruck, Motion of level sets by mean curvature III, Journal of Geometric Analysis 2 (1992), 121-150. MR 93d:58044

[ESIV] L. C. Evans and J. Spruck, Motion of level sets by mean curvature IV, Journal of Geometric Analysis 5 (1995), 77-113. MR 96a:35077

[ESS] L.C. Evans, H.M. Soner, and P.E. Souganidis, Phase transitions and generalized motion by mean curvature, Comm. Pure Appl. Math 45 (1992), 1097-1123. MR 93g:35064

[HayPomm] W. K. Hayman and Ch. Pommerenke, On analytic functions of bounded mean oscillation, Bulletin of the London Mathematical Society 10 (1978), 219-224. MR 81g:30044

[Hunt] G. A. Hunt, Markoff processes and potentials. III, Illinois J. Math. 2 (1958), 151-213. MR 21:5824 
[Illmanen] T. Ilmanen, Convergence of the Allen-Cahn equation to Brakke's motion by mean curvature, J. Differential Geom. 38 (1993), 417-461. MR 94h:58051

[KS] I. Karatzas and S. Shreve, Brownian Motion and Stochastic Calculus (2nd ed.), Springer, New York, 1991. MR 92h:60127

[Mueller] C. Mueller, A characterization of $B M O$ and $B M O_{\rho}$, Studia Mathematica 72 (1982), 47-57. MR 84j:42032

[RY] D. Revuz, and M. Yor, Continuous Martingales and Brownian Motion, SpringerVerlag, New York, 1991. MR 92d:60053

[Sonera] H. M. Soner, Ginzburg-Landau equation and motion by mean curvature, I: convergence, (to appear), Journal of Geometric Analysis.

[Sonerb] H. M. Soner, Ginzburg-Landau equation and motion by mean curvature, II: development of the interface, (to appear), Journal of Geometric Analysis.

[Stegenga] D. A. Stegenga, A geometric condition which implies BMOA, Michigan Mathematical Journal 27 (1980), 247-252. MR 81h:30039

[Stroock] D. W. Stroock, Probability Theory, An Analytic View, Cambridge University Press, New York, 1993. MR 95f:60003

[Watson] N. A. Watson, Thermal capacity, Proc. London Mathematical Society (3) 37 (1978), 342-362. MR 80b:31005

Department of Mathematics, University of Illinois at Urbana-Champaign, Urbana, ILLINOIS 61801-2917

E-mail address: r-sowers@math.uiuc.edu

Department of Mathematics, University of Illinois at Urbana-Champaign, Urbana, ILLINOIS 61801-2917

E-mail address: wu@math.uiuc.edu 